\title{
Standpunkt
}

Gegenentwürfe zur derzeitigen Wirtschaftsweise

\section{Für einen Angewandten Utopismus}

\author{
Utopien beschreiben andere Verhältnisse, um reale \\ Gesellschaften zu kritisieren oder Alternativen zu ihnen \\ zu entwerfen. Sie können ernst sein oder spielerisch. \\ Alle Formen und Realitätsbezüge des Utopischen \\ haben ihren Ort. Aber wir möchten für eine bestimmte \\ Spielart plädieren: Angewandten Utopismus. \\ Von Roland Bluhm und Cornelia Auer
}

D ie Wissenschaft weist uns auf Entwicklungen hin, die in verschiedene dystopische Szenarien führen können. Das sind zunächst ökologische Katastrophen wie der Klimawandel, das Artensterben und die Erschöpfung beziehungsweise die Verschmutzung der natürlichen Ressourcen. Dazu gehören aber auch Probleme der Verteilungsgerechtigkeit, wie Armut und extreme Ungleichheit, die durch den Klimawandel in Zukunft noch verstärkt werden dürften.

Diesen existenziellen Gefahren und moralischen Unzumutbarkeiten können wir nur begegnen, indem wir unsere Gesellschaften in kürzester Zeit umfassend und tiefgreifend transformieren - insbesondere unsere Wirtschaftsweise, eine zentrale Ursache dieser Entwicklungen. Daher liegt es nahe, nach utopischen Gegenentwürfen zu suchen, die radikal genug wären, die Ursachen unserer Lage anzugehen.

\section{Radikal andere Wirtschafts- formen denken}

Wo über radikal andere Wirtschaftsformen nachgedacht wird, findet man zurzeit zwei gegensätzliche Lager: Das eine (zu dem etwa Kate Raworth zählt) bietet eher freundliche Einladungen, entwickelt Konzepte, die nicht auf konkrete Handlungsempfehlungen und die hierfür erforderlichen Instrumente fokussiert sind. Gegebenenfalls wird es kommenden Generationen anvertraut, eine
Evolution der Verhältnisse zu vollziehen. Das andere Lager zeigt sich offensiver, ist näher an der Anwendung und kann beispielsweise vorrechnen, warum es sich sozial und ökonomisch lohnt, schnellstmöglich eine andere Wirtschaftsweise zu verwirklichen. Meist handelt es sich um Ausnahmeerscheinungen (wie etwa Joseph E. Stiglitz), auch weil es für die Arbeit an radikalen Modellen, die den Status quo angreifen, seltener Forschungsgelder gibt.

\section{Utopismus voranbringen}

Braucht der Utopismus also anderes Personal? Nein, wir glauben, dass sich nicht die Utopist/innen selbst ändern müssen, sondern ihre Arbeitsweise. Neben der allgemeinen Moralphilosophie oder Ethik hat sich in den letzten Jahrzehnten die sogenannte Angewandte Ethik entwickelt. Sie untersucht Bereiche menschlichen Handelns, die sich nicht befriedigend mithilfe allgemeiner ethischer Theorien reflektieren lassen, sondern die die Mühen der Auseinandersetzung mit komplexen empirischen Begebenheiten erfordern. Ohne sie bliebe die ethische Reflexion zu allgemein, zu unkonkret, unterkomplex. Der Utopismus muss einen ähnlichen Schritt machen.

Angewandter Utopismus ist kooperativ und professionell, nicht Geniekult. Er ist realistisch und pragmatisch, entwirft also umsetzbare Visionen und will sie auch umsetzen. Er ist integrativ, in- sofern er sich für Teilhabe öffnet. Und er ist keine Angelegenheit der üblichen Verdächtigen, beschränkt sich auch nicht auf die Perspektive von Ökonom/innen, sondern bindet Akteur/innen aus Transformationsfeldern ein, um eine angemessene Komplexität zu erreichen: Energieversorgung, Logistik, Militär, Industrie, Handel und so weiter.

\section{Umsetzbare Visionen entwickeln}

Dabei verleugnen sich die Utopist/innen nicht, indem sie etwa bodenständigere Ideen $\mathrm{zu}$ formulieren versuchen, sondern sie begeben sich in einen Raum, in dem ihre Vorstellungen und die der Anwender/innen eine Spannung halten, die sie weder in spekulativen Unmöglichkeiten noch in Bestandsdenken erstarren lassen.

Diese Spannung ermöglicht die Entstehung realistischer Ideen für die massiven Transformationen, die eine nachhaltige Zukunft erfordert.

AUTOR/INNEN + KONTAKT

Dr. Roland Bluhm ist Geschäftsführer der DBG Dr Bluhm GmbH (Außenhandel mit Umweltmesstechnik) und Dozent

für gute wissenschaftliche Praxis.

DBG Dr Bluhm GmbH, Oppelner Str. 30, 10997 Berlin. Tel.: +49 3042093294 ,

E-Mail: roland.bluhm@drbluhmgmbh.com, Internet: www.drbluhmgmbh.com

Dr. Cornelia Auer ist Wissenschaftlerin am Potsdam-Institut für Klimafolgenforschung (PIK) e. V. Ihr Arbeitsfeld sind Mitigationsszenarien und Klimafolgenprojektionen.

Potsdam-Institut für Klimafolgenforschung (PIK) e. V., Postfach 601203,14412 Potsdam. Tel.: +49 331 288-20787,

E-Mail: cornelia.auer@pik-potsdam.de, Internet: www.pik-potsdam.de 